\title{
A Closer Look at the Behavior of Uncertainty and Disagreement Micro Evidence from the Euro Area
}

Robert Rich and J oseph Tracy

Federal Reserve Bank of Dallas Research Department Working Paper 1811

https://doi.org/10.24149/wp1811 


\title{
A Closer Look at the Behavior of Uncertainty and Disagreement: Micro Evidence from the Euro Area
}

\author{
Robert Rich \\ Joseph Tracy*
}

July 24, 2018

\begin{abstract}
This paper examines point and density forecasts of real GDP growth, inflation and unemployment from the European Central Bank's Survey of Professional Forecasters. We present individual uncertainty measures and introduce individual point- and density-based measures of disagreement. The data indicate substantial heterogeneity and persistence in respondents' uncertainty and disagreement, with uncertainty associated with prominent respondent effects and disagreement associated with prominent time effects. We also examine the co-movement between uncertainty and disagreement and find an economically insignificant relationship that is robust to changes in the volatility of the forecasting environment. This provides further evidence that disagreement is not a reliable proxy for uncertainty.
\end{abstract}

Keywords: Disagreement; Uncertainty; Point Forecasts; Density Forecasts; Heterogeneity; ECB Survey of Professional Forecasters

JEL Codes: C10, C12, C23

\footnotetext{
* Rich: Federal Reserve Bank of New York; Tracy: Federal Reserve Bank of Dallas. The authors thank Ken West and Stefania D'Amico as well as conference participants at the Fall 2017 System Committee meeting and the 5 $5^{\text {th }}$ Workshop on Empirical Macroeconomics for very helpful comments. Michael Stewart, Michael Fosco, Daniel Reuter and Max Sterman provided excellent research assistance. Corresponding author: Robert Rich. The views expressed in the paper are those of the authors and do not necessarily reflect those of the Federal Reserve Bank of New York or the Federal Reserve System. Address for correspondence: Research and Statistics Group, 33 Liberty Street, Federal Reserve Bank of New York, NY 10045-0001. Phone: 212 720-8100. Email: robert.rich@,ny.frb.org
} 


\section{Introduction}

Individuals' forecast behavior remains an area of considerable research interest because of its importance for understanding decision-making, as well as for explaining movements in economic and financial variables. The majority of studies have focused on expectations, but other dimensions of forecast behavior such as disagreement and uncertainty also play an important role in macroeconomic analysis. ${ }^{1}$ Despite their importance, the measurement of disagreement and uncertainty - like the measurement of expectations - is challenging because of the inherent difficulty in observing individuals' subjective assessments. While many surveys offer information about point forecasts and their dispersion across respondents, most typically do not provide information about the degree of confidence that respondents attach to their point forecasts.

There are, however, a limited number of surveys that provide both point and density forecasts, with the latter taking the form of subjective probability distributions. The availability of density forecasts allows for a more detailed analysis of forecast behavior than can be carried out using point forecasts alone. For example, density forecasts provide a basis to construct measures of individual and aggregate uncertainty. In addition, studies using density forecasts have documented substantial heterogeneity and persistence in forecasters' uncertainty.

This paper examines point and density forecasts from the European Central Bank's Survey of Professional Forecasters (ECB-SPF) that solicits euro area expectations for real GDP growth, a harmonized index of consumer prices (HICP) inflation and the unemployment rate. In particular, we use the ECB-SPF data for an empirical investigation into three issues related to the measurement and behavior of uncertainty and disagreement. First, we construct individual measures of uncertainty and disagreement, with the latter involving the introduction of new respondent-specific measures of disagreement derived from both point and density forecasts. By using metrics of the divergence between two distributions, we extend the notion of disagreement beyond its conventional association with differences in point forecasts to include, as well, differences in density forecasts. These new measures of forecast dispersion allow us to undertake a parallel analysis of disagreement and uncertainty, as well as to gauge the robustness of the results to the alternative forecast dispersion measures.

\footnotetext{
${ }^{1}$ For example, it is argued that uncertainty is an important source of business cycle fluctuations [Bloom (2009), Bloom et al. (2018)] and a fundamental determinant of asset prices [Campbell (2000)]. Differences in agents' expectations have been cited as an important channel for monetary policy to affect real activity [Woodford (2003), Mankiw and Reis (2002), Lorenzoni (2009)] and a key factor influencing the effect of public information signals [Morris and Shin (2002), Amador and Weill (2010)].
} 
Second, we analyze statistical features of the individual measures of uncertainty and disagreement across the three forecast variables. Specifically, we examine the cross-sectional behavior of uncertainty and disagreement, as well as their movements over time. Moreover, and in contrast to studies conducted at the aggregate level, we consider the roles of respondent and time effects in the behavior of uncertainty and disagreement. This provides a useful background for our formal investigation into the issues of heterogeneity and persistence.

Last, we investigate the relationship between individual uncertainty and disagreement. Numerous empirical studies_-lacking a direct measure of aggregate uncertainty-have used aggregate disagreement as a proxy for aggregate uncertainty under the maintained assumption of a meaningful positive association between the two variables. Uncertainty and disagreement, however, are distinct concepts and theory offers little guidance about their association. Moreover, while the conceptual distinction between uncertainty and disagreement is clear at the individual level, direct measurement and testing of their association has, to date, been entirely at the aggregate level. Because aggregation may obscure the true relationship, the forecast dispersion measures in this study allow us to evaluate the reliability of disagreement as a proxy for uncertainty at the individual level. Given that the global financial crisis falls approximately in the middle of our sample period, we investigate whether there is a change in the linkage between disagreement and uncertainty associated with this event.

The empirical analysis yields several findings of note. There is substantial heterogeneity in forecasters' uncertainty and disagreement. In addition, individual uncertainty is associated with prominent respondent effects, while individual disagreement is associated with prominent time effects. Thus, differences across respondents' measured uncertainty feature a pronounced timeinvariant element that is consistent with our finding that uncertainty displays higher persistence than disagreement. Moreover, the differential presence of time-invariant and time-dependent elements in individual uncertainty and disagreement is consistent with our finding of an economically insignificant co-movement between the series. Further, this conclusion is unaffected when we consider sub-samples before and after the global financial crisis that we associate, respectively, with a tranquil and volatile forecasting environment. Taken together, our findings at the individual level corroborate the majority of evidence at the aggregate level that disagreement is not a reliable proxy for uncertainty.

The paper is organized as follows. The next section provides a summary of the literature focusing on survey-based measures of uncertainty and disagreement. The third section describes the ECB-SPF data. Section four introduces the individual measures of uncertainty and disagreement, as 
well as investigates statistical features of the series. The section also details the regression models used to analyze the co-movement between disagreement and uncertainty and reports the statistical and economic significance of the estimated relationship. The final section concludes by discussing the implications of our findings.

\section{Literature Review}

While there are a large number of surveys that report point forecasts, only a small number also provide density forecasts. These include the ECB-SPF examined here, as well as the U.S. Survey of Professional Forecasters (US-SPF), the Bank of England Survey of External Forecasters (BOESEF) and the Federal Reserve Bank of New York Survey of Consumer Expectations (FRBNYSCE).

An issue of central importance to our analysis is the measurement of uncertainty and disagreement. Several different methods have been used to measure uncertainty based on the reported density (histogram) forecasts. In some cases, the uncertainty measure is derived from the individual density forecasts assuming that the probability mass within each interval is concentrated at the midpoint of each interval [Rich and Tracy (2010), Kenny et al. (2015)], or that the probability mass is distributed uniformly within each interval [Zarnowitz and Lambros (1987), Abel et al. (2016)]. ${ }^{2}$ In other cases, the uncertainty measure is derived by fitting a continuous distribution to the individual density forecasts, where the continuous distribution is a normal distribution [Giordani and Soderlind (2003), Boero et al. (2014)], a generalized beta distribution [Bruine de Bruin et al. (2011)], or both [Clements (2014a, 2014b)]. ${ }^{3}$ Alternatively, other studies [Batchelor and Dua (1996), Boero et al. (2008)] have focused on constructing an aggregate density forecast and then using a decomposition of the aggregate variance to derive a measure of average uncertainty.

To date, aggregate disagreement has been measured using the dispersion of respondents' point forecasts as reflected in the cross-sectional variance/standard deviation, the interquartile range (IQR), or the quasi-standard deviation (qsd). ${ }^{4}$ While there are a few studies that have used density forecasts to derive a measure of disagreement, they have focused on the dispersion of individual density forecast means [Giordani and Soderlind (2003), Boero et al. (2008), D'Amico and

\footnotetext{
2 D'Amico and Orphanides (2008) derive measures of uncertainty under both assumptions.

${ }^{3}$ As noted by Bruine de Bruin et al. (2011), Clements (2014a, 2014b) and Boero et al. (2014), reported nonzero probabilities are needed in at least three bins to allow fitting to proceed. They follow the approach of Engelberg et al. (2009) and construct uncertainty measures by fitting triangular distributions when a respondent assigns probabilities to two intervals or less.

${ }^{4}$ In this case, the quasi-standard deviation is equal to half the difference between the $16^{\text {th }}$ and $84^{\text {th }}$ percentiles of the sample of point forecasts appropriately interpolated.
} 
Orphanides (2008), Bruine de Bruin et al. (2011)]. As we discuss in the next section, our measures of disagreement differ from previous measures in two important dimensions. First, we derive the measures at the individual level rather than at the aggregate level. Second, we implement them for both point and density forecasts. This is important because the density-based measure of disagreement is more general than the point-based measure of disagreement as it encompasses any differences in the density forecasts, rather than just in their first moments.

Another area of interest concerns the characteristics of disagreement and uncertainty. In addition to examining the behavior of disagreement about point forecasts and density forecast means, studies have explored heterogeneity in forecasters' uncertainty [D'Amico and Orphanides (2008), Boero et al. (2008), Bruine de Bruin et al. (2011), Clements (2014b), Boero et al. (2014)]. The finding of substantial heterogeneity in reported point forecasts and density forecasts has resulted in further exploration into the behavior over time of disagreement and uncertainty. There is evidence of persistent differences in respondents' point forecasts [Patton and Timmermann (2010), Boero et al. (2014)], as well as in respondents' forecast uncertainty [Bruine de Bruin et al. (2011), Boero et al. (2014)].

A number of studies have also explored the relationship between disagreement and uncertainty, but the existing empirical evidence has only been at the aggregate level and has relied mainly on data from the US-SPF. The evidence from the US-SPF on this relationship has been mixed. Zarnowitz and Lambros (1987) report a modest positive association between disagreement and uncertainty. Giordani and Soderlind (2003) extend the sample period of Zarnowitz and Lambros and report a positive association between disagreement and uncertainty that is both economically and statistically significant, although some studies have argued that their conclusion is problematic. ${ }^{5}$ Examining matched point and density forecasts, Rich and Tracy (2010) find a very weak relationship between disagreement and uncertainty for the US-SPF. Recent analyses that have examined data from other surveys featuring point and density forecasts, such as Boero et al. (2008) for the BOE-SEF and Abel et al. (2016) for the ECB-SPF, have found little support for the use of disagreement as proxy for uncertainty. ${ }^{6}$

\footnotetext{
${ }^{5}$ Rich and Tracy (2010) and Boero et al. (2014) discuss the problematic nature of fitting normal distributions to histograms where respondents place positive probability in less than three bins.

${ }^{6}$ Boero $e t$ al. (2014) find a strong positive correlation between disagreement and uncertainty when they extend their 2008 analysis of the BOE-SEF to include the recent crisis period. However, the results are driven principally by observations in the series associated with the initial onset of the crisis. The authors conclude that disagreement appears to be a useful proxy for uncertainty when it exhibits large fluctuations, but low-level high frequency variations in the series are not sufficiently correlated.
} 
In an interesting paper, Lahiri and Sheng (2010) argue that the disagreement-uncertainty relationship may be episodic. They develop a Bayesian learning model in which agents hold diverse prior beliefs and receive a public signal about the forecasted target variable, where the precision of the public signal can change over time. In their framework, greater uncertainty in the interpretation of the public signal decreases its information content and results in disagreement reflecting relatively more of the diversity in forecasters' prior beliefs. On the other hand, lower uncertainty in the interpretation of the public signal raises its information content. Consequently, forecasters will attach greater weight to the public signal, which, assuming they interpret the public signal in a similar manner, will lead to lower disagreement. An implication of their model is that the strength of the disagreement-uncertainty relationship will be inversely related to the volatility of the forecasting environment. Because our sample period includes the global financial crisis, it provides an opportunity to examine if the relationship between disagreement and uncertainty is sensitive to the change in volatility related to this event.

\section{The European Central Bank's Survey of Professional Forecasters}

Since 1999, the ECB-SPF provides a quarterly survey of forecasts for euro area real GDP growth, the harmonized index of consumer price (HICP) inflation and unemployment. The ECBSPF asks panelists for forecasts at short-, medium- and longer-term horizons including both rolling and calendar year variants. The ECB-SPF draws its pool of panelists from both financial and nonfinancial institutions throughout the euro area, with a little more than 40 panelists responding on average per survey. For additional details about the ECB-SPF, see Garcia (2003) and Bowles et al. (2007).

For the three macroeconomic variables — real GDP growth, inflation and unemploymentthe ECB-SPF asks respondents to submit both a point and a density forecast. For the density forecasts, respondents provide a probability distribution of forecasted outcomes. Specifically, they report their probability distribution as a histogram using a set of intervals provided by the ECB. The ECB-SPF changes over time the number of closed intervals for the histogram in an effort to limit the likelihood that respondents place either a significant probability or no probability in either of the two open intervals. However, the ECB-SPF uses a common width for the closed intervals and this width has remained fixed. ${ }^{7}$

\footnotetext{
7 This contrasts with the US-SPF that has experienced periodic changes in interval widths of the density forecasts.
} 
In our analysis, we examine matched point and density forecasts for real GDP growth, HICP inflation and the unemployment rate that involve a "rolling" one-year-ahead horizon. ${ }^{8}$ Given that the horizon length remains constant through time, we treat the data as quarterly observations on a set of homogenous series. As Garcia (2003) notes, there is a temporal misalignment between the target variables because of differences in the data frequency and publication lags of the variables. Specifically, real GDP growth is published quarterly with a two-quarter lag, while HICP inflation and the unemployment rate and are published monthly with a one-month lag and a two-month lag, respectively. ${ }^{9}$

With regard to the sample, our study covers the period 1999Q1-2017Q3. For each survey and forecast variable, we exclude any respondent who does not report matched point and density forecasts, or whose probabilities for a density forecast do not sum to unity. In addition, we exclude the 2009Q1 real GDP growth density forecast data because many respondents in this survey placed significant probability in the lower open interval of their real GDP growth density forecasts. ${ }^{10}$

Before turning to the empirical analysis, there is a caveat that merits attention relating to the density forecasts reported by the ECB-SPF or similar surveys. As discussed in Boero et al. (2014), it is important to recognize that any reported probability distribution will be an imperfect representation of the respondent's forecast uncertainty. They note the apparent difficulty of forecasters to give precise numerical values for their subjective probabilities. In addition, the limited number of intervals to which many respondents assign probabilities precludes the specification of a parametric probability distribution, with any fitted distribution necessarily expressing uncertainty about the target variable in more detail than the forecaster has conveyed. Consequently, researchers should view a survey-based measure of uncertainty as an estimated proxy for the respondent's unobserved uncertainty, and some caution should be exercised when interpreting results and making inferences about uncertainty and its features based on this proxy.

\footnotetext{
${ }^{8}$ There are also forecasts reported for the a one-year/one-year forward horizon and a longer calendar horizon - four-calendar years ahead for surveys conducted in the Q1 and Q2 rounds, and five-calendar years ahead for surveys conducted in the Q3 and Q4 rounds.

${ }^{9}$ For example, the 2010Q1 survey questionnaire asks respondents to forecast one-year-ahead output growth from 2009Q3-2010Q3. For HICP inflation, the corresponding forecast horizon is December 2009December 2010. For the unemployment rate, the corresponding forecast is for November 2010.

${ }^{10}$ For this survey, the significant probability mass at the lower open interval corresponded to a growth rate of " $-1 \%$ or less" and was due to the survey design of the density forecasts and its inability to provide sufficient coverage for the pessimistic point predictions of output growth. For individuals who either reported point predictions below $-1 \%$ or wanted to indicate significant downside risk, they assigned most of their probability to the open-ended interval. The narrow spread of the probabilities across intervals results in an artificially low value of the uncertainty measure. See Abel et al. (2016) for further discussion.
} 


\section{Variable Definitions}

The traditional survey-based disagreement measure used in the literature is either the crosssectional variance or standard deviation of the point forecasts. If we let ${ }_{i} f_{t}^{e}$ denote the point forecast from respondent $i$ in the survey at date $t, n_{t}$ the number of respondents in the survey, and $\bar{f}_{t}^{e}$ the mean of these point forecasts, then the variance-based aggregate disagreement measure is given by:

$$
D_{t}=\frac{1}{n_{t}-1} \sum_{i=1}^{n_{t}}\left({ }_{i} f_{t}^{e}-\bar{f}_{t}^{e}\right)^{2}
$$

The mean point forecast is also typically referred to as the "consensus" forecast.

Generalizing, we can define an individual "average point disagreement” (APD) measure for the $j^{\text {th }}$ respondent as the average squared distance between this respondent's point forecast and the other respondents' point forecasts:

$$
{ }_{j} A P D_{t}=\frac{1}{n_{t}-1} \sum_{i \neq j}\left({ }_{i} f_{t}^{e}-{ }_{j} f_{t}^{e}\right)^{2}
$$

The traditional variance-based aggregate disagreement measure can be thought of as the individual APD measure for a representative respondent whose point forecast coincides with the consensus mean.

The APD measure uses the squared norm to measure the distance between two respondents' point forecasts. However, we can also construct an individual disagreement measure using the absolute value norm, which places less weight on large pair-wise differences. Consequently, there is a robustness consideration associated with the choice of which distance norm to use. Specifically, measures based on the squared norm are more sensitive to outliers compared to measures based on the absolute value norm. We will point out in a subsequent discussion that uncertainty measures can be sensitive to the treatment of the open intervals of the density forecasts, with the sensitivity again being higher when using a squared norm. Because the absolute value norm helps to mitigate both of 
these concerns, we adopt this metric for all distance calculations. ${ }^{11}$ Accordingly, we define the individual average absolute point disagreement (AAPD) measure as:

$$
{ }_{j} A A P D_{t}=\frac{1}{n_{t}-1} \sum_{i \neq j}\left|{ }_{i} f_{t}^{e}-{ }_{j} f_{t}^{e}\right|
$$

The traditional aggregate disagreement measure and individual disagreement measures just discussed are based on respondents' point forecasts. For surveys like the ECB-SPF that report respondents' density forecasts, we can construct additional individual-level disagreement measures that reflect more information than is revealed in the point forecasts. To illustrate this conceptually, consider two respondents $i$ and $j$, where we fix the distance between their point forecasts across two scenarios, but vary the nature of the density forecasts. Assume that each respondent's point forecast corresponds to the mid-point of the bin of their density forecast containing the highest probability. Consider the contrasting sets of densities in Figure 1. In scenario A, respondent $i$ 's density forecast and respondent $j$ 's density forecast are skewed to the right. In Scenario B, respondent $i$ 's density forecast does not change, while respondent $j$ 's density forecast is now skewed to the left and away from respondent $i$ 's point forecast. The point forecasts in each scenario are the same, and therefore would indicate the same degree of disagreement. However, based on the additional information conveyed by the density forecasts, it is reasonable to argue that the degree of disagreement between the two respondents is larger in scenario B. ${ }^{12}$

What we need, then, is a metric to convey the degree of divergence between two histograms. Following the choice of the absolute value metric to calculate dispersion of the point forecasts, we use the Wasserstein distance measure for histograms. ${ }^{13}$ Let ${ }_{i} F_{t}^{-1}$ denote the inverse cumulative density function (CDF) for respondent $i$ in the survey at date $t$, where we assume the probability mass is distributed uniformly within each bin of the histogram. The Wasserstein disagreement (WD) measure between respondent $i$ and respondent $j$ is given by:

\footnotetext{
11 While the subsequent analysis restricts its attention to measures constructed using the absolute value norm, we also repeated the regression analysis with measures constructed using the squared norm. The results were qualitatively similar and are available in a separate Results Appendix to conserve on space.

12 To further illustrate this point consider two density forecasts where one density is changed using a meanpreserving spread - disagreement measured by the point forecasts or the density means would not change, while disagreement based on the density forecasts would change.

13 See Arroyo and Mate (2009). The Wasserstein measure is also referred to as the earth mover's distance.
} 


$$
{ }_{i j} W D_{t}=\int_{0}^{1}\left|{ }_{i} F_{t}^{-1}(z)-{ }_{j} F_{t}^{-1}(z)\right| d z
$$

We then define the individual average absolute density disagreement (AADD) measure for respondent $j$ as follows:

$$
{ }_{j} A A D D_{t}=\frac{1}{n_{t}-1} \sum_{i \neq j}{ }_{i j} W D_{t}
$$

Our density-based measure of dispersion has several attractive features. First, the measure uses the same density forecasts as the uncertainty measure, potentially providing a closer linkage between movements in the series. Second, the measure allows us to capture any differences across density forecasts by focusing on the whole cumulative distribution function rather than specific moments. Consequently, the density-based disagreement measure may be more informative than its point-based counterpart.

Turning to uncertainty, a popular proxy is the variance of a survey respondent's density forecast. Zarnowitz and Lambros (1987) examine the US-SPF and derive the variance assuming a uniform distribution within each interval. The US-SPF density forecast, like that for the ECB-SPF, contains open intervals on each end of the histogram that must be closed to calculate this variance. A typical —although ad hoc-assumption is to close these exterior open intervals by assigning them twice the width of the interior closed intervals. After closing off the two open intervals, assume that there are $k_{t}$ bins associated with the histogram, that the upper and the lower values for the $i^{\text {th }}$ bin are given, respectively, by $\boldsymbol{u}_{i}$ and $l_{i}$, and that the probability assigned by respondent $j$ to this bin is ${ }_{j} p_{t}^{i}$. The probability distribution variance (PDV) measure of individual uncertainty is then given by:

$$
{ }_{j} P D V_{t}=\left[\sum_{i=1}^{k_{t}}{ }_{j} p_{t}^{i}\left(\frac{u_{i t}^{3}-l_{i t}^{3}}{3\left(u_{i t}-l_{i t}\right)}\right)\right]-\left[\sum_{i=1}^{k_{t}}{ }_{j} p_{t}^{i}\left(\frac{u_{i t}^{2}-l_{i t}^{2}}{2\left(u_{i t}-l_{i t}\right)}\right)\right]^{2}
$$

To the extent that respondents place any probability in either open interval, the manner chosen to close off the open intervals will affect a variance measure of uncertainty. In an attempt to mitigate this concern, our analysis uses instead the inner quartile range (IQR) to proxy uncertainty. This measure will be robust to the treatment of the open intervals as long as respondents do not place more than 25 percent probability in either open interval. While most 
forecasters only assign probabilities to the interior closed intervals, in almost every quarter there are forecasters who assign probability to the open intervals. Moreover, the number of forecasters assigning probability to the open intervals can occasionally be notable. However, forecasters rarely place more than 25 percent probability in an open interval, thereby making the IQR an attractive alternative to the variance. ${ }^{14} \mathrm{We}$ calculate the IQR uncertainty measure $\left({ }_{j} I Q R_{t}\right)$ assuming, as we did for the WD measure and the variance, that the probability mass is distributed uniformly within each bin of the histogram.

\section{A closer look at the point- and density-based measures of disagreement}

Because the individual point- and density based measures of disagreement provide new metrics of forecast dispersion, it is useful to examine and compare some of their properties as background before undertaking a more detailed investigation into their behavior and that of uncertainty. Figure 2 shows scatterplots of each respondent's point- and density-based measures of disagreement for the three forecast variables over the full sample period along with a 45-degree reference line. There are several important takeaways from the scatterplots. The first is that, absent any shape differences in the density forecasts, both disagreement measures are the same. To understand this, consider the following simple example of two respondents whose density forecasts are identical except for their location. Assume that the point forecast for each respondent corresponds to the implied median of their respective density forecasts. In this example, all percentiles of the density forecasts differ by the same amount as the medians. An implication is the difference between the inverse CDFs in equation (4) will always equal the point disagreement value, so that the subsequent integration yields a density disagreement that is equal to the point disagreement. Consequently, divergences between a respondent's point and density disagreement measures reflect shape differences between the density forecasts.

A second observation from Figure 2 is that the density- and point-based measures of disagreement display an asymmetry at low levels of forecast dispersion. Specifically, at levels of point disagreement less than 0.5, a disproportionate share of the dots are above the 45-degree line indicating the density-based measures of disagreement tend to be higher than their point-based counterparts. However, the asymmetry essentially disappears at higher levels of disagreement as the dots are more evenly distributed on either side of the 45-degree line. This suggests that the density

\footnotetext{
${ }^{14}$ This occurred in less than 1 percent of the surveys. For real GDP growth, inflation and unemployment, the percentages were $0.54 \%$ [ 17 responses out of 3,164], $0.03 \%$ [ 1 response out of 3,309], and $0.81 \%$ [ 24 responses out of 2,962], respectively.
} 
disagreement measure tends to identify relatively more disagreement than the point disagreement measure in environments of low of disagreement.

A third finding relates to the important question of how much independent variation there is in the density disagreement measures controlling for the point disagreement measures. When we regressed the density disagreement on the point disagreement, the $R^{2}$ value ranges from 0.63 to 0.76. However, the correlation between the two disagreement measures changes dramatically as the level of forecast dispersion rises. For each of the three forecast variables, we used the median value of the point-based disagreement measure to partition the data and re-ran the regressions of the density disagreement on the point disagreement for each of the two samples. For the regressions involving the lower disagreement levels, the $R^{2}$ value ranged from 0.04 to 0.20 . In contrast, for the higher disagreement levels, the $R^{2}$ value ranged from 0.62 to 0.80 . So, while location of the densities as captured by the point disagreement generates the larger source of overall disagreement variation, shape differences in the densities adds additional information particularly at lower levels of disagreement. As a result, to the extent that these two disagreement measures differ in their relationship with other variables, that difference likely arises in low disagreement situations.

\section{Heterogeneity and persistence}

Drawing upon the literature that has examined heterogeneity and persistence in various features of forecast behavior, we undertake a similar analysis for our measures of disagreement and uncertainty. Figure 3 and Figure 4 plot, respectively, the distributions of our individual disagreement and uncertainty measures for the three forecast variables. ${ }^{15}$ The distributions allow us to see the cross-sectional spread in the individual-level measures, as well as their behaviors over time. As a point of reference, the green line in each panel depicts the median value, around which we observe considerable dispersion, implying substantial heterogeneity across respondents in their degrees of disagreement and uncertainty. In addition, the distributions typically display positive skewness. Note that the vertical scale of the disagreement measures for real GDP growth and inflation is different from that of the uncertainty measures.

\footnotetext{
${ }^{15}$ Figure 3 and Figure 4 plot values for the real GDP growth forecasts from the 2009Q1 survey for completeness. As noted earlier, we exclude these values from the regression analysis because of problems associated with the reported density forecasts.
} 
All of the disagreement measures spike during the global financial crisis, with a subsequent smaller spike occurring with the onset of the European debt crisis. ${ }^{16}$ While the individual uncertainty measures in Figure 4 display a greater degree of cross-sectional dispersion compared to the disagreement measures, they display less of a spike during the global financial crisis. Instead, the spreads of the uncertainty measures have increased since the crisis as the upper-half of the individual uncertainty distributions have moved higher from that time and have yet to revert to their pre-crisis levels. In addition, and perhaps more importantly, the uncertainty measures display less variability than the disagreement measures. This feature of the data is particularly relevant for our subsequent variance decompositions and regression analysis.

While Figure 3 and Figure 4 depict the manner in which the distributions of individual disagreement and uncertainty shift over time, they do not indicate the degree to which respondents move over time within the distributions. In particular, they do not indicate whether there are persistent patterns in individual forecasters' disagreement or uncertainty and, if so, the sources for such persistence. To address the former issue, we draw upon Patton and Timmermann (2010) and provide in Tables 1-3 quarterly transition matrices for the three forecast variables, where we rank all respondents according to the quartile in which their measures of disagreement and uncertainty fall in each survey. In the absence of persistence in forecasters' relative disagreement and uncertainty, the entries in the tables should all be approximately one-quarter (0.25). If, however, there is persistence in these features of forecast behavior, then terms on the diagonal should be significantly higher than 0.25 , and consequently the off-diagonal terms should be smaller. For each table, we provide the sample transition rates and examine if differences in forecasters' relative disagreement and uncertainty persist using the relevant one-tailed test. We also report the chi-square statistic from a joint test for the entire table following a uniform distribution.

Taken together, there is very strong evidence of persistence for the disagreement and uncertainty measures as indicated by the large number of statistical tests rejecting the relevant null hypothesis. For all three forecast variables, there is also more evidence of persistence in the densitybased disagreement measure than in the point-based disagreement measure. In particular, all of the estimated probabilities of remaining in the same quartile for the real GDP growth and inflation density-based disagreement measures are significantly greater than 25 percent. For the individual uncertainty measures, there is even greater persistence as shown by three of the four diagonal

\footnotetext{
16 As another point of reference, the traditional aggregate disagreement measure corresponds approximately to the $0^{\text {th }}$ percentile of the individual disagreement measures as the sample mean (across the other respondents) by construction minimizes the value of equation (1).
} 
probabilities being above 50 percent for all three forecast variables. In the case of persistence in individual uncertainty, our results are consistent with those reported by Boero et al. (2014) for the BOE-SEF and by Bruine de Bruin et al. (2011) for the FRBNY-SCE. The evidence of persistence in individual disagreement is a new finding, although evidence of persistence in the relative level of point forecasts has been documented by Patton and Timmermann (2010) for the US-SPF and by Boero et al. (2014) for the BOE-SEF.

The evidence of persistence in the individual disagreement and uncertainty measures motivates our exploration into the sources for the persistence. Here we will consider the role of respondent effects that reflect any systematic differences across respondents that are unrelated to the surveys in which they participate. That is, some respondents may be inherently more or less uncertain, or may consistently display higher or lower disagreement than others. In estimating the contribution of respondent effects, we will first control for any time effects to account for the fact that collectively respondents' uncertainty or disagreement may vary over time. Controlling for time effects is important because not all respondents participate in all surveys. ${ }^{17}$

Table 4 provides variance decompositions for each measure across the three forecast variables. As shown, the presence of time-dependent and time-invariant elements differs markedly across the disagreement and uncertainty measures. For the two disagreement measures, time effects account for a significant portion of the overall variance, while respondent effects are much less important. Across the three forecast variables, the disagreement time effects are largest for real GDP growth. Uncertainty, in contrast, has a much larger component of its variance explained by respondent effects than by time effects. ${ }^{18}$ The relative importance of the respondent effects is also fairly similar for the uncertainty measure across the three forecast variables. Taken together, the results in Table 4 underscore the importance of accounting for respondent and time effects in our subsequent regression analysis. In particular, the evidence that respondents' measured uncertainty and personal disagreement feature, respectively, a prominent time-invariant element and prominent common temporal element is consistent with our findings that uncertainty displays higher persistence and less variability than disagreement.

\footnotetext{
17 That is, the ECB-SPF, like other surveys, is an unbalanced panel as it has experienced exit and entry of respondents over time and occasional non-response to the complete questionnaire. The current analysis uses the unbalanced panel structure because the inclusion of respondent fixed effects and time fixed effects allows us to control for possible compositional effects.

${ }^{18}$ Bruine de Bruin et al. (2011) also document that there is a strong fixed effect component associated with individual respondents' uncertainty.
} 


\section{Regression Models and Empirical Results}

In situations where researchers only have access to point forecasts, there may be an interest in using a point forecast disagreement measure as a proxy for respondent uncertainty. The validity of disagreement as a proxy for uncertainty depends critically on disagreement having a strong empirical association with uncertainty controlling for other explanatory variables in the specification.

To investigate the relationship between disagreement and uncertainty, we consider the following two regression models that allow us to examine the point- and density forecast data at the individual level:

$$
\begin{aligned}
& { }_{j} I Q R_{t}=\beta_{0}^{P}+\beta_{1}^{P}\left({ }_{j} A A D D_{t}\right)+\alpha_{j}^{P}+\mu_{t}^{P}+{ }_{j} \varepsilon_{t}^{P} \\
& { }_{j} I Q R_{t}=\beta_{0}^{D}+\beta_{1}^{D}\left({ }_{j} A A D D_{t}\right)+\alpha_{j}^{D}+\mu_{t}^{D}+{ }_{j} \varepsilon_{t}^{D}
\end{aligned}
$$

where $\alpha_{j}$ denotes a respondent fixed effect, $\mu_{t}$ a time fixed effect, and ${ }_{j} \varepsilon_{t}$ a mean-zero random disturbance term. While the motivation above relies only on the relationship between point forecast dispersion and uncertainty, we consider measures of disagreement derived from both the point and density forecasts. Consequently, the notation in the regression models will differentiate the disagreement measures by the superscripts ' $\mathrm{P}$ ' and ' $\mathrm{D}$ '. We estimate the two relationships using the unbalanced panel data of respondents, and we calculate the standard errors by clustering at the respondent level. Given the assumed positive association between disagreement and uncertainty, we conduct a one-sided test of statistical significance for $\beta_{1}$.

Table 5 presents simple regression results for real GDP growth, inflation and the unemployment rate that consider both of our individual point- and density-based disagreement measures. In each case, we show results using the overall variance of each disagreement variable (specifications (1) and (4)), next controlling for the time-series component of the disagreement variance (specifications (2) and (5)), and then finally controlling for both the time-series and respondent components of the disagreement variance (specifications (3) and (6)). In each case, we indicate the incremental $R^{2}$ value from adding the indicated disagreement measure to the other variables in the regression.

Focusing first on the point-based disagreement measures, there is a positive and statistically significant relationship between individual uncertainty and individual disagreement for real GDP growth and inflation using the overall variation in disagreement. However, the estimated 
relationships explain little of the overall variation of individual uncertainty, as the reported $R^{2}$ values are very small. Moreover, the few instances of statistical significance are not robust to including time and respondent effects, which also result in incremental $R^{2}$ values that are essentially zero.

Turning to the density-based disagreement measures, there is a stronger and more robust positive relationship between individual uncertainty and individual disagreement. Given the lack of explanatory power of the point disagreement measures, the explanatory power from the density disagreement measures is due to the independent variation in these density disagreement measures that is concentrated at low levels of forecast dispersion as we documented earlier. That is, movements in the density disagreement measures at low levels of forecast dispersion, which principally reflect changes in the shape of the density forecasts, are able to pick up corresponding movements in uncertainty. Nevertheless, while the statistical significance of the relationship is robust to including time and respondent effects, the incremental explanatory content of disagreement is again modest and progressively declines as we control for the time- and respondentspecific components of the variance. ${ }^{19}$ Consequently, the initial results for the ECB-SPF at the individual level support the finding of Abel et al. (2016) at the aggregate level that there is no meaningful association between uncertainty and disagreement. This result would not seem surprising based on the earlier analysis documenting the different statistical features of the series at the individual level.

\section{The uncertainty-disagreement relationship and the forecasting environment}

The results presented in Table 5 are based on regression model estimates for the variables of interest over the period 1999Q1-2017Q3. As previously noted, there is evidence of increased uncertainty over the post-2007 period that, based on the analysis of Lahiri and Sheng (2010), raises the issue of possible instability in the uncertainty-disagreement relationship. In addition, there are notable spikes in personal disagreement over the post-2007 period. Taken together, these considerations argue for the need to investigate if the previous finding of an absence of a meaningful association between uncertainty and disagreement is robust to changes in the volatility of the forecasting environment.

\footnotetext{
${ }^{19}$ For real GDP growth and unemployment, variation in the density disagreement measures due to respondent effects account for the largest share of its overall explanatory power. For inflation, variation in the density disagreement measure due to time effects accounts for the largest share of its overall explanatory power.
} 
To address this issue, we divide the full sample period into two sub-sample periods by excluding the observations for 2008 and 2009. Tables 6a-b present the regression results for each of the three forecast variables and for our individual point- and density-based disagreement measures over the 1999Q1-2007Q4 and 2010Q1-2017Q3 sub-periods, respectively. Because the 2010Q12017Q3 sub-period reflects higher uncertainty, we characterize the associated forecasting environment in this sub-period as more volatile.

Comparing the two sub-periods, the results appear to be qualitatively similar. The pointbased measures of individual disagreement are generally statistically insignificant and often display an inverse relationship with uncertainty, with the latter feature particularly evident over the 2010Q12017Q3 sub-period. In contrast, the density-based measures of individual disagreement display a statistically significant positive association with uncertainty that is robust to including time and respondent effects. With regard to the predictions of Lahiri and Sheng's (2010) Bayesian learning model, we find mixed evidence of a lower estimated coefficient on disagreement during the more volatile sub-period. Moreover, and likely of greater importance, the incremental explanatory content of disagreement is modest in each sub-period, and differences in the estimated marginal impact of disagreement on uncertainty do not appear to be either economically or statistically significant across the sub-periods. ${ }^{20}$ Taken together, the results from the sub-period analysis indicate that the absence of a meaningful relationship between disagreement and uncertainty is not a consequence of variability in the forecasting environment. That is, the failure of disagreement to serve as a reliable proxy for uncertainty appears to be a general result.

\section{Conclusion}

This paper uses data on reported point and density forecasts from the ECB-SPF to present individual measures of uncertainty and introduce individual point and density-based measures of disagreement. We analyze the statistical properties of these two aspects of forecast behavior, as well as assess their co-movement. In terms of motivation and contribution, our study complements the larger literature that has focused on the measurement of expectations. In particular, the expanded scope of our investigation serves as a basis for a better understanding and improved formulation of the beliefs formation process of individuals.

\footnotetext{
${ }^{20} \mathrm{We}$ also considered an alternative approach in which model estimation used data from the full sample period, but allowed for a one-time shift in the coefficient on the disagreement measure over the 2008Q12017Q3 sub-period. The results supported the conclusion drawn from Tables 6a-b. Specifically, formal statistical testing failed to reject the null hypothesis of no change in the coefficient on personal disagreement across the two sub-periods at conventional levels of significance.
} 
Our empirical analysis indicates substantial heterogeneity and persistence in respondents’ uncertainty and personal disagreement, although there are also notable differences in their features. Uncertainty is associated with strong respondent effects, while personal disagreement is associated with strong time effects, with movements in the level of uncertainty displaying higher persistence. We also document that the association between the point- and density-based measures of disagreement strengthens as the level of forecast dispersion increases. Moreover, the scope for independent variation in the point- and density-based measures of disagreement is sufficient to allow for possible differences in the estimation results.

With regard to the co-movement between uncertainty and disagreement, we find evidence indicating that there is a notable difference in the nature of the relationship between uncertainty and the point- and density-based measures of disagreement. Specifically, there is a stronger as well as more robust positive and statistically significant relationship between individual uncertainty and individual density-based disagreement measures. However, the magnitude of the correlation between uncertainty and disagreement is quite small, suggesting movements in the variables are largely independent of each other. Moreover, the findings are robust to the incidence of the global financial crisis. Similar to conclusions drawn using data at the aggregate level, our results at the individual level do not support the practice of using disagreement as a proxy for uncertainty.

Taken together, our findings lead to suggestions for further research. One issue of interest is identifying differences in the nature or impact of the underlying factors guiding the divergent behavior of uncertainty and disagreement. Another issue relates to the strong persistence displayed by uncertainty during a sample period that contains both a tranquil and volatile episode, with the latter including the recent global financial crisis. The availability of measures of uncertainty and disagreement at the individual level would allow us to investigate whether heterogeneity in these forecast aspects results in heterogeneity in forecast behavior. It would also be possible to explore the role of disagreement and uncertainty in the forecast revision process of respondents. Developing theoretical models that can account for the features described above would offer a significant advancement in the study of the beliefs formation process. 


\section{References}

Abel, Joshua, Robert Rich, Joseph Song, and Joseph Tracy. "The Measurement and Behavior of Uncertainty: Evidence from the ECB Survey of Professional Forecasters." Journal of Applied Econometrics 31 (April/May 2016): 533-550. https://doi.org/10.1002/jae.2430

Arroyo, Javier and Carlos Mate. "Forecasting Histogram Time Series with K-Nearest Neighbours Mehods.” International Journal of Forecasting 25 (January-March 2009): 192-207. https://doi.org/10.1016/j.ijforecast.2008.07.003

Amador, Manuel, and Pierre-Oliver Weill. "Learning From Prices: Public Communication and Welfare." Journal of Political Economy 118 (October 2010): 866-907. https://doi.org/10.1086/657923

Batchelor, Roy S., and Pami Dua. "Empirical Measures of Inflation Uncertainty: A Cautionary Note." Applied Economics 28 (March 1996): 333-341. https://doi.org/10.1080/000368496328704

Bloom, Nicholas. "The Impact of Uncertainty Shocks." Econometrica 77 (May 2009): 623-685. https://doi.org/10.3982/ecta6248

Bloom, Nicholas, Max Floetotto, Nir Jaimovich, Saporata-Eksten, Terry Itay, and J. Stephen. "Really Uncertain Business Cycles." Econometrica 86 (May 2018): 1031-1065. https://doi.org/10.3982/ecta10927

Boreo, Gianna, Jeremy Smith, and Kenneth F. Wallis. "Uncertainty and Disagreement in Economic Prediction: The Bank of England Survey of External Forecasters." Economic Journal 118 (July 2008): 1107-1127. https://doi.org/10.1111/j.1468-0297.2008.02162.x

Boero, Gianna, Jeremy Smith, and Kenneth F. Wallis. "The Measurement and Characteristics of Professional Forecasters' Uncertainty." Journal of Applied Econometrics 30 (November/December 2014): 1029-1046. https://doi.org/10.1002/jae.2400

Bowels, Carlos, Roberta Friz, Veronique Genre, Geoff Kenny, Aidan Meyler, and Tuomas Rautanen. “The ECB Survey of Professional Forecasters (SPF): A Review After Eight Years' Experience.” ECB Occasional Paper No. 59, April 2007.

Bruine de Bruin, Wandi, Charles F. Manski, Giorgio Topa, and Wilbert van der Klaauw. "Measuring Consumer Uncertainty About Future Inflation." Journal of Applied Econometrics 26 (April-May 2011): 454-478. https://doi.org/10.1002/jae.1239

Campbell, John Y. "Asset Pricing at the Millennium." Journal of Finance 55 (August 2000): 1515-1567. https://doi.org/10.1111/0022-1082.00260

Clements, Michael P. "Evaluating the Bank of England Density Forecasts of Inflation." The Economic Journal 114 (October 2004): 844-866. https://doi.org/10.1111/j.1468-0297.2004.00246.x -. "Probability Distributions or Point Predictions? Survey Forecasts of US Output Growth and Inflation." International Journal of Forecasting 30 (January-March 2014a): 99-117. https://doi.org/10.1016/i.ijforecast.2013.07.010

-----. "Forecast Uncertainty_Ex Ante and Ex Post: U.S. Inflation and Output Growth." Journal of Business and Economic Statistics 32 (April 2014b): 206-216. https://doi.org/10.1080/07350015.2013.859618

Diebold, Francis X., Anthony S. Tay, and Kenneth F. Wallis. "Evaluating Density Forecasts of Inflation: The Survey of Professional Forecasters." In Cointegration, Causality, and Forecasting: A Festschrift in Honour of Clive W. J. Granger, edited by Robert F. Engle and Halbert White, 7690. Oxford and New York, Oxford University Press, 1999. https://doi.org/10.3386/w6228

D'Amico, Stefania, and Athanasios Orphanides. "Uncertainty and Disagreement in Economic Forecasting." Finance and Economics Discussion Series 2008-56. Federal Reserve Board, Washington DC, 2008. https://doi.org/10.2139/ssrn.2853147 
Engelberg, Joseph, Charles F. Manski, and Jared Williams. "Comparing the Point Predictions and Subjective Probability Distributions of Professional Forecasters.” Journal of Business and economic Statistics 27 (January 2009): 30-41. https://doi.org/10.1198/jbes.2009.0003

Garcia, Juan A. "An Introduction to the ECB's Survey of Professional Forecasters." ECB Occasional Paper No 8, September 2003.

Giordani, Paolo, and Paul Soderlind. "Inflation Forecast Uncertainty." European Economic Review 47 (December 2003): 1037-1059. https://doi.org/10.1016/s0014-2921(02)00236-2

Kenny, Geoff, Thomas Kostka, and Federico Masera. "How Informative are the Subjective Density Forecasts of Macroeconomists?" Journal of Forecasting 33 (April 2014): 163-185. https://doi.org/10.1002/for.2281

------, Thomas Kostka, and Federico Masera. "Density Characteristics and Density Forecast Performance: A Panel Analysis." Empirical Economics 48 (May 2015): 1203-1231. https://doi.org/10.1007/s00181-014-0815-9

Lahiri, Kajal, and Xuguang Sheng. "Measuring Forecast Uncertainty by Disagreement: The Missing Link." Journal of Applied Econometrics 25 (June/July 2010): 514-538. https://doi.org/10.1002/jae.1167

Lorenzoni, Guido. "A Theory of Demand Shocks." Journal of Political Economy 99 (December 2009): 2050-2084. https://doi.org/10.3386/w12477

Mankiw, Gregory N., and Ricardo Reis. "Sticky Information Versus Sticky Prices: A Proposal to Replace the New Keynesian Phillips Curve." Quarterly Journal of Economics 117 (November 2002): 1295-1328. https://doi.org/10.1162/003355302320935034

Morris, Stephen, and Hyun Song Shin. "Social Value of Public Information." American Economic Review 92 (December 2002): 1521-1534.

Patton, Andrew J., and Allan Timmermann. "Why Do Forecasters Disagree? Lessons From the Term Structure of Cross-Sectional Dispersion." Journal of Monetary Economics 57 (October 2010): 803-820. https://doi.org/10.1016/j.jmoneco.2010.07.001

Rich, Robert W., and Joseph Tracy. "The Relationship Among Expected Inflation, Disagreement, and Uncertainty: Evidence From Matched Point and Density Forecasts." Review of Economics and Statistics 92 (February 2010): 200-207. https://doi.org/10.1162/rest.2009.11167

Woodford, Michael. "Imperfect Common Knowledge and the Effects of Monetary Policy." In Knowledge, Information, and Expectations in Modern Macroeconomics: In Honor of Edmund S. Phelps, edited by Philippe Aghion, Roman Frydman, Joseph Stiglitz and Michael Woodford. Princeton, Princeton University Press, 2003. https://doi.org/10.3386/w8673

Zarnowitz, Victor, and Louis A. Lambros. "Consensus and Uncertainty in Economic Predictions." Journal of Political Economy 95 (June 1987): 591-621. https://doi.org/10.3386/w1171 
Table 1. Transition Probabilities - Real GDP Growth

a) Average Absolute Point Disagreement

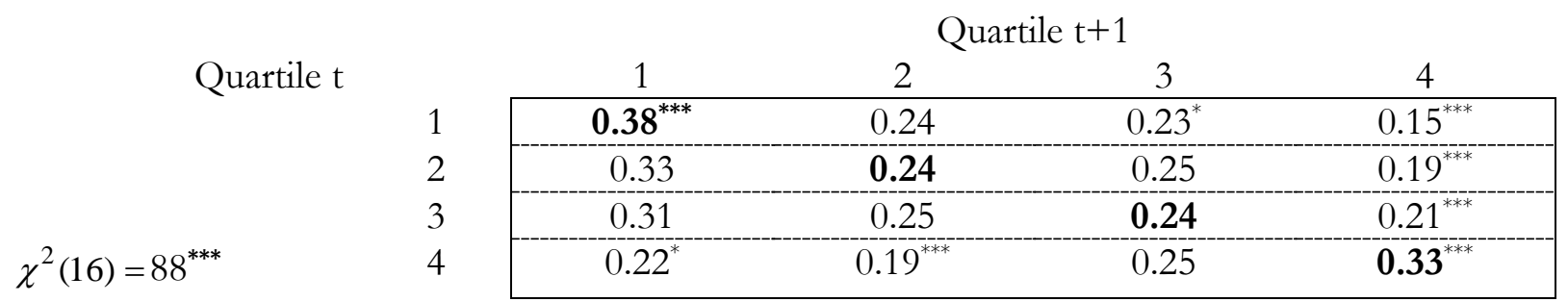

b) Average Absolute Density Disagreement

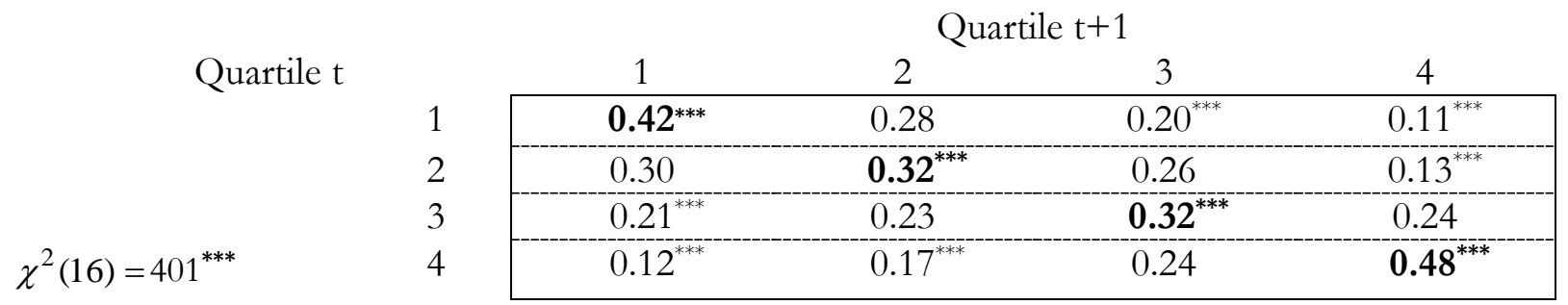

c) Uncertainty (IQR)

Quartile t

$\chi^{2}(16)=2,140^{* * *}$

\begin{tabular}{|c|c|c|c|}
\hline \multicolumn{4}{|c|}{ Quartile $\mathrm{t}+1$} \\
\hline 1 & 2 & 3 & 4 \\
\hline $0.66^{* * *}$ & 0.26 & $0.06^{* * *}$ & $0.02^{* * *}$ \\
\hline 0.30 & $0.46^{\text {*** }}$ & $0.20^{* * * *}$ & $0.03^{* * *}$ \\
\hline $0.06^{* * *}$ & $0.20^{\text {***** }}$ & $0.55^{\text {*** }}$ & $0.19^{* * *}$ \\
\hline $0.01^{* * *}$ & $0.04^{* * *}$ & $0.20^{* * *}$ & $0.75^{* * *}$ \\
\hline
\end{tabular}

Notes: One-tailed tests for individual diagonal (off-diagonal) elements $>(<) 0.25$.

Chi-square statistics are for a joint test for uniform distribution for the entire table.

${ }^{* * *}$ significant at the $1 \%$ level

** significant at the $5 \%$ level

* significant at the $10 \%$ level 
Table 2. Transition Probabilities - Inflation

a) Average Absolute Point Disagreement

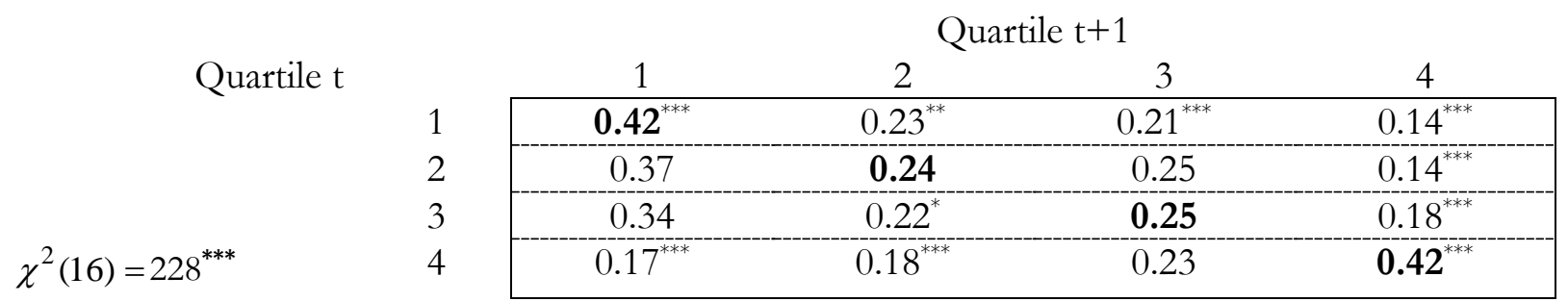

b) Average Absolute Density Disagreement

\begin{tabular}{|c|c|c|c|c|c|}
\hline & & & & & \\
\hline & & 1 & 2 & 3 & 4 \\
\hline & 1 & $0.47^{* * *}$ & 0.27 & $0.16^{* * *}$ & $0.10^{* * *}$ \\
\hline & 2 & 0.29 & $0.31^{* * *}$ & 0.23 & $0.17^{* * *}$ \\
\hline & 3 & $0.18^{* * *}$ & 0.25 & $0.36^{\text {**** }}$ & $0.21^{\text {*** }}$ \\
\hline$\chi^{2}(16)=518^{* * *}$ & 4 & $0.12^{* * *}$ & $0.14^{* * *}$ & 0.26 & $0.48^{* * *}$ \\
\hline
\end{tabular}

c) Uncertainty (IQR)

Quartile t

$\chi^{2}(16)=2,310^{* * *}$

\begin{tabular}{|cccc|}
\multicolumn{5}{c}{1} & \multicolumn{2}{c}{ Quartile $\mathrm{t}+1$} & \\
\hline $\mathbf{0 . 6 6}$ & 2 & 3 & 4 \\
\hline 0.28 & 0.25 & $0.08^{* * *}$ & $0.01^{* * *}$ \\
\hline $0.08^{* * *}$ & $\mathbf{0 . 4 6 ^ { * * * * }}$ & 0.23 & $0.03^{* * *}$ \\
\hline $0.02^{* * *}$ & $0.21^{* * * *}$ & $\mathbf{0 . 5 6 ^ { * * * }}$ & $0.16^{* * *}$ \\
\hline $0.05^{* * * * *}$ & $0.17^{* * *}$ & $\mathbf{0 . 7 7 ^ { * * * }}$ \\
\hline
\end{tabular}

Notes: One-tailed tests for individual diagonal (off-diagonal) elements $>(<) 0.25$.

Chi-square statistics are for a joint test for uniform distribution for the entire table.

*** significant at the $1 \%$ level

** significant at the $5 \%$ level

${ }^{*}$ significant at the $10 \%$ level 
Table 3. Transition Probabilities - Unemployment

a) Average Absolute Point Disagreement

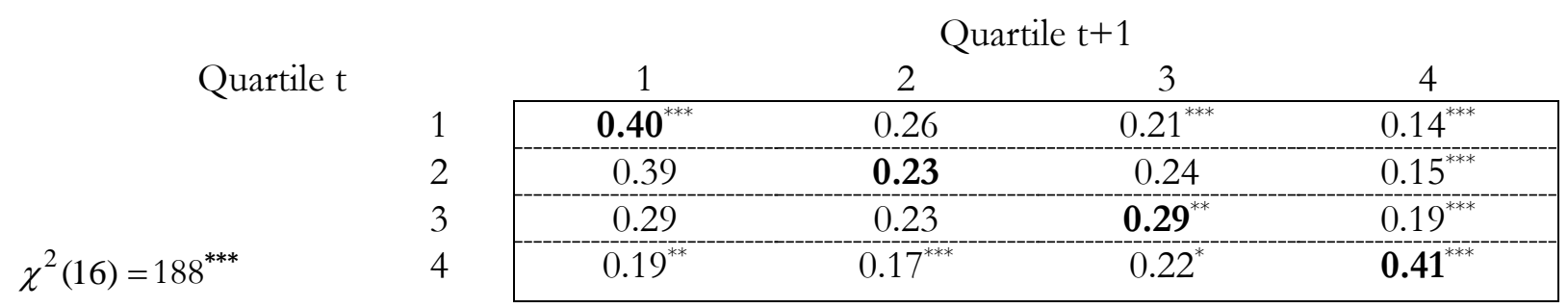

b) Average Absolute Density Disagreement

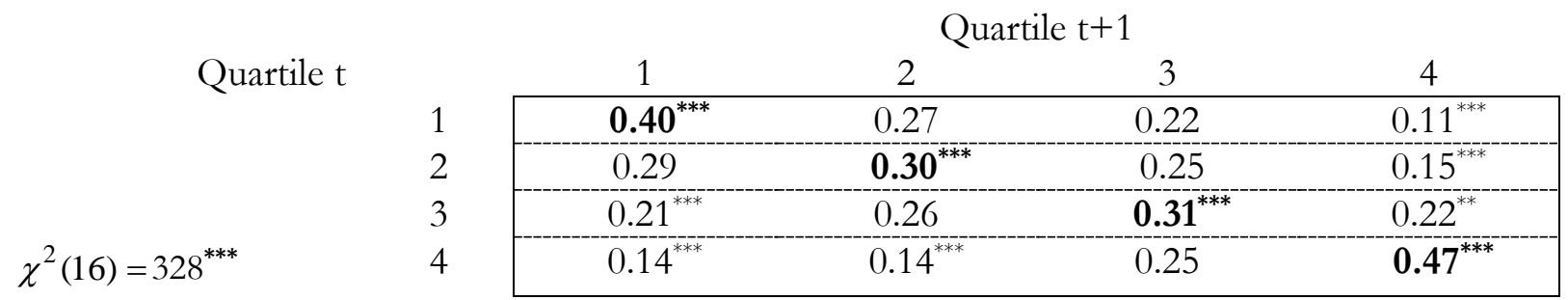

c) Uncertainty (IQR)

Quartile t

$\chi^{2}(16)=1,801^{* * *}$

\begin{tabular}{|c|c|c|c|}
\hline \multicolumn{4}{|c|}{ Quartile $t+1$} \\
\hline 1 & 2 & 3 & 4 \\
\hline $0.62^{* * *}$ & 0.28 & $0.08^{* * *}$ & $0.02^{* * *}$ \\
\hline 0.31 & $0.44^{* * *}$ & $0.20^{* * *}$ & $0.04^{* * *}$ \\
\hline $0.09^{* * *}$ & $0.21^{* *}$ & $0.52^{* * *}$ & $0.18^{* * *}$ \\
\hline $0.02^{* * *}$ & $0.06^{* * *}$ & $0.18^{* * *}$ & 0.74 \\
\hline
\end{tabular}

Notes: One-tailed tests for individual diagonal (off-diagonal) elements $>(<) 0.25$.

Chi-square statistics are for a joint test for uniform distribution for the entire table.

*** significant at the $1 \%$ level

** significant at the $5 \%$ level

${ }^{*}$ significant at the $10 \%$ level 
Table 4. Variance Decompositions

a) Average Absolute Point Disagreement

\begin{tabular}{rccc} 
Source & Real GDP Growth & Inflation & Unemployment \\
\hline Time & $44.0 \%$ & $28.7 \%$ & $36.2 \%$ \\
Respondent & $5.7 \%$ & $8.9 \%$ & $7.7 \%$ \\
Residual & $49.3 \%$ & $62.0 \%$ & $55.6 \%$
\end{tabular}

b) Average Absolute Density Disagreement

\begin{tabular}{rccc} 
Source & Real GDP Growth & Inflation & Unemployment \\
\hline Time & $44.9 \%$ & $31.5 \%$ & $31.5 \%$ \\
Respondent & $7.7 \%$ & $10.4 \%$ & $9.7 \%$ \\
Residual & $47.0 \%$ & $57.4 \%$ & $57.9 \%$
\end{tabular}

c) Uncertainty (IQR)

\begin{tabular}{rccc} 
Source & Real GDP Growth & Inflation & Unemployment \\
\hline Time & $8.0 \%$ & $10.8 \%$ & $11.7 \%$ \\
Respondent & $47.7 \%$ & $45.8 \%$ & $39.2 \%$ \\
Residual & $39.2 \%$ & $37.8 \%$ & $45.5 \%$ \\
\hline
\end{tabular}

Notes: ECB-SPF data. Authors' calculations. 
Table 5. Relationship of Uncertainty (IQR) to Disagreement: 1999Q1-2017Q3

\section{a) Real GDP Growth}

$$
\text { (1) }
$$

(3)

(4)

(5)

(6)

\begin{tabular}{lcccccc} 
Average Absolute & $0.107 * * *$ & $0.078^{* *}$ & 0.014 & & & \\
Point Disagreement & $(0.033)$ & $(0.040)$ & $(0.028)$ & & & \\
Average Absolute & & & & $0.392^{* * *}$ & $0.475^{* * *}$ & $0.225^{* * *}$ \\
Density Disagreement & & & & $(0.078)$ & $(0.126)$ & $(0.071)$ \\
Constant & $0.684^{* * *}$ & $0.569^{* * *}$ & $0.579^{* * *}$ & $0.552^{* * *}$ & $0.419^{* * *}$ & $0.507 * * *$ \\
& $(0.032)$ & $(0.041)$ & $(0.032)$ & $(0.028)$ & $(0.048)$ & $(0.038)$ \\
\hline Time effects & $\mathrm{N}$ & $\mathrm{Y}$ & $\mathrm{Y}$ & $\mathrm{N}$ & $\mathrm{Y}$ & $\mathrm{Y}$ \\
Respondent effects & $\mathrm{N}$ & $\mathrm{N}$ & $\mathrm{Y}$ & $\mathrm{N}$ & $\mathrm{N}$ & $\mathrm{Y}$ \\
Incremental R $\mathrm{R}^{2}$ for disagreement & 0.006 & 0.002 & 0.001 & 0.073 & 0.058 & 0.012
\end{tabular}

b) Inflation

\begin{tabular}{|c|c|c|c|c|c|c|}
\hline & $(1)$ & (2) & (3) & (4) & $(5)$ & (6) \\
\hline Average Absolute & $0.114 * * *$ & -0.001 & 0.013 & & & \\
\hline Point Disagreement & $(0.043)$ & $(0.053)$ & $(0.038)$ & & & \\
\hline Average Absolute & & & & $0.386^{* * *}$ & $0.265^{* * *}$ & $0.153^{* * *}$ \\
\hline Density Disagreement & & & & $(0.075)$ & $(0.085)$ & $(0.056)$ \\
\hline Constant & $\begin{array}{c}0.648^{* * *} \\
(0.026)\end{array}$ & $\begin{array}{c}0.554 * * * \\
(0.037)\end{array}$ & $\begin{array}{c}0.609 * * * \\
(0.026)\end{array}$ & $\begin{array}{c}0.543 * * * \\
(0.021)\end{array}$ & $\begin{array}{c}0.449 * * * \\
(0.038)\end{array}$ & $\begin{array}{c}0.553^{* * *} \\
(0.035)\end{array}$ \\
\hline Time effects & $\mathrm{N}$ & $\mathrm{Y}$ & $\mathrm{Y}$ & $\mathrm{N}$ & $\mathrm{Y}$ & $\mathrm{Y}$ \\
\hline Respondent effects & $\mathrm{N}$ & $\mathrm{N}$ & Y & $\mathrm{N}$ & $\mathrm{N}$ & Y \\
\hline Incremental $\mathrm{R}^{2}$ for disagreement & 0.005 & 0.000 & 0.000 & 0.048 & 0.017 & 0.005 \\
\hline c) Unemployment & $(1)$ & (2) & (3) & (4) & $(5)$ & (6) \\
\hline Average Absolute & 0.054 & -0.052 & -0.043 & & & \\
\hline Point Disagreement & $(0.037)$ & $(0.055)$ & $(0.032)$ & & & \\
\hline Average Absolute & & & & $0.521 * * *$ & $0.536^{* * *}$ & $0.349 * * *$ \\
\hline Density Disagreement & & & & $(0.100)$ & $(0.136)$ & $(0.104)$ \\
\hline Constant & $\begin{array}{c}0.647 * * * \\
(0.031) \\
\end{array}$ & $\begin{array}{c}0.562 * * * \\
(0.037)\end{array}$ & $\begin{array}{c}0.571 * * * \\
(0.027)\end{array}$ & $\begin{array}{c}0.458^{* * *} \\
(0.031)\end{array}$ & $\begin{array}{c}0.309 * * * \\
(0.053)\end{array}$ & $\begin{array}{c}0.423^{* * *} \\
(0.048) \\
\end{array}$ \\
\hline Time effects & $\mathrm{N}$ & $\mathrm{Y}$ & $\mathrm{Y}$ & $\mathrm{N}$ & $\mathrm{Y}$ & $\mathrm{Y}$ \\
\hline Respondent effects & $\mathrm{N}$ & $\mathrm{N}$ & $\mathrm{Y}$ & $\mathrm{N}$ & $\mathrm{N}$ & Y \\
\hline Incremental $\mathrm{R}^{2}$ for disagreement & 0.001 & 0.001 & 0.001 & 0.098 & 0.070 & 0.026 \\
\hline
\end{tabular}

Notes: Standard errors are reported in parentheses and are based on clustering at the respondent level. The incremental $\mathrm{R}^{2}$ pertains to the disagreement variable. For specifications (1) and (4), the incremental $\mathrm{R}^{2}$ is identical to the overall $\mathrm{R}^{2}$.

*** significant at the 1\% level

** significant at the 5\% level

* significant at the 10\% level 
Table 6a. Relationship of Uncertainty (IQR) to Disagreement: 1999Q1-2007Q4

a) Real GDP Growth

(1)

(2)

(3)

(4)

(5)

(6)

\begin{tabular}{|c|c|c|c|c|c|c|}
\hline $\begin{array}{l}\text { Average Absolute } \\
\text { Point Disagreement }\end{array}$ & $\begin{array}{c}0.114 * * * \\
(0.043)\end{array}$ & $\begin{array}{c}0.115^{* *} \\
(0.058)\end{array}$ & $\begin{array}{c}0.034 \\
(0.032)\end{array}$ & & & \\
\hline Average Absolute & & & & $0.409 * * *$ & $0.517 * * *$ & $0.290 * * *$ \\
\hline Density Disagreement & & & & $(0.133)$ & $(0.171)$ & $(0.110)$ \\
\hline Constant & $\begin{array}{c}0.592^{* * *} \\
(0.028) \\
\end{array}$ & $\begin{array}{c}0.557 * * * \\
(0.043) \\
\end{array}$ & $\begin{array}{c}0.649 * * * \\
(0.029) \\
\end{array}$ & $\begin{array}{c}0.470^{* * *} \\
(0.041) \\
\end{array}$ & $\begin{array}{c}0.403^{* * *} \\
(0.058) \\
\end{array}$ & $\begin{array}{c}0.569^{* * *} \\
(0.042) \\
\end{array}$ \\
\hline Time effects & $\mathrm{N}$ & $\mathrm{Y}$ & $\mathrm{Y}$ & $\mathrm{N}$ & $\mathrm{Y}$ & $\mathrm{Y}$ \\
\hline Respondent effects & $\mathrm{N}$ & $\mathrm{N}$ & $\mathrm{Y}$ & $\mathrm{N}$ & $\mathrm{N}$ & Y \\
\hline Incremental $\mathrm{R}^{2}$ & 0.006 & 0.004 & 0.000 & 0.070 & 0.078 & 0.020 \\
\hline \multicolumn{7}{|l|}{ b) Inflation } \\
\hline & $(1)$ & (2) & (3) & (4) & (5) & (6) \\
\hline Average Absolute & -0.009 & 0.029 & 0.045 & & & \\
\hline Point Disagreement & $(0.066)$ & $(0.073)$ & (0.053) & & & \\
\hline Average Absolute & & & & $0.301 * * *$ & $0.441 * * *$ & $0.283^{* * *}$ \\
\hline Density Disagreement & & & & $(0.103)$ & $(0.122)$ & $(0.085)$ \\
\hline \multirow[t]{2}{*}{ Constant } & $0.593^{* * *}$ & $0.544^{* * *}$ & $0.737 * * *$ & $0.493^{* * *}$ & $0.380^{* * *}$ & $0.641 * * *$ \\
\hline & $(0.029)$ & $(0.042)$ & $(0.026)$ & $(0.026)$ & $(0.043)$ & $(0.038)$ \\
\hline Time effects & $\mathrm{N}$ & $\mathrm{Y}$ & $\mathrm{Y}$ & $\mathrm{N}$ & $\mathrm{Y}$ & $\mathrm{Y}$ \\
\hline Respondent effects & $\mathrm{N}$ & $\mathrm{N}$ & $\mathrm{Y}$ & $\mathrm{N}$ & $\mathrm{N}$ & $\mathrm{Y}$ \\
\hline \multirow[t]{3}{*}{ Incremental R² } & 0.000 & 0.000 & 0.000 & 0.028 & 0.042 & 0.008 \\
\hline & & & & & & \\
\hline & $(1)$ & (2) & (3) & (4) & (5) & (6) \\
\hline Average Absolute & -0.035 & -0.018 & 0.005 & & & \\
\hline Point Disagreement & $(0.057)$ & $(0.069)$ & $(0.040)$ & & & \\
\hline Average Absolute & & & & $0.425^{* * *}$ & $0.540^{* * *}$ & $0.343^{* * *}$ \\
\hline Density Disagreement & & & & $(0.130)$ & $(0.163)$ & $(0.091)$ \\
\hline \multirow[t]{2}{*}{ Constant } & $0.579 * * *$ & $0.549 * * *$ & $0.676^{* * *}$ & $0.420^{* * *}$ & $0.307 * * *$ & $0.542^{* * *}$ \\
\hline & $(0.027)$ & $(0.038)$ & $(0.034)$ & $(0.036)$ & $(0.063)$ & $(0.052)$ \\
\hline Time effects & $\mathrm{N}$ & $\mathrm{Y}$ & $\mathrm{Y}$ & $\mathrm{N}$ & $\mathrm{Y}$ & $\mathrm{Y}$ \\
\hline Respondent effects & $\mathrm{N}$ & $\mathrm{N}$ & $\mathrm{Y}$ & $\mathrm{N}$ & $\mathrm{N}$ & Y \\
\hline Incremental $\mathrm{R}^{2}$ & 0.000 & 0.000 & 0.000 & 0.072 & 0.094 & 0.030 \\
\hline
\end{tabular}

Notes: Standard errors are reported in parentheses and are based on clustering at the respondent level. The incremental $R^{2}$ pertains to the disagreement variable. For specifications (1) and (4), the incremental $\mathrm{R}^{2}$ is identical to the overall $\mathrm{R}^{2}$.

*** significant at the $1 \%$ level

${ }^{* *}$ significant at the $5 \%$ level

* significant at the $10 \%$ level 
Table 6b. Relationship of Uncertainty (IQR) to Disagreement: 2010Q1-2017Q3

a) Real GDP Growth

$(2)$

(3)

(4)

(5)

(6)

\begin{tabular}{lcccccc} 
Average Absolute & 0.045 & 0.064 & -0.027 & & & \\
Point Disagreement & $(0.074)$ & $(0.088)$ & $(0.062)$ & & & \\
Average Absolute & & & & $0.578^{* * *}$ & $0.780^{* * *}$ & $0.182^{* *}$ \\
Density Disagreement & & & & $(0.169)$ & $(0.228)$ & $(0.108)$ \\
Constant & $0.834^{* * *}$ & $0.825^{* * *}$ & $0.718^{* * *}$ & $0.591^{* * *}$ & $0.407 * * *$ & $0.586^{* * *}$ \\
& $(0.054)$ & $(0.071)$ & $(0.057)$ & $(0.067)$ & $(0.138)$ & $(0.078)$ \\
\hline Time effects & $\mathrm{N}$ & $\mathrm{Y}$ & $\mathrm{Y}$ & $\mathrm{N}$ & $\mathrm{Y}$ & $\mathrm{Y}$ \\
Respondent effects & $\mathrm{N}$ & $\mathrm{N}$ & $\mathrm{Y}$ & $\mathrm{N}$ & $\mathrm{N}$ & $\mathrm{Y}$ \\
Incremental R & 0.001 & 0.001 & 0.000 & 0.078 & 0.100 & 0.004
\end{tabular}

b) Inflation

\begin{tabular}{lcccccc} 
& $(1)$ & $(2)$ & $(3)$ & $(4)$ & $(5)$ & $(6)$ \\
\hline Average Absolute & -0.009 & -0.027 & -0.024 & & & \\
Point Disagreement & $(0.069)$ & $(0.087)$ & $(0.053)$ & & & \\
Average Absolute & & & & $0.180^{* *}$ & $0.199^{*}$ & 0.072 \\
Density Disagreement & & & & $(0.105)$ & $(0.129)$ & $(0.084)$ \\
Constant & $0.812^{* * *}$ & $0.840^{* * *}$ & $0.678^{* * *}$ & $0.736^{* * *}$ & $0.741^{* * *}$ & $0.636^{* * *}$ \\
& $(0.042)$ & $(0.059)$ & $(0.046)$ & $(0.035)$ & $(0.058)$ & $(0.055)$ \\
\hline Time effects & $\mathrm{N}$ & $\mathrm{Y}$ & $\mathrm{Y}$ & $\mathrm{N}$ & $\mathrm{Y}$ & $\mathrm{Y}$ \\
Respondent effects & $\mathrm{N}$ & $\mathrm{N}$ & $\mathrm{Y}$ & $\mathrm{N}$ & $\mathrm{N}$ & $\mathrm{Y}$ \\
Incremental R & 0.000 & 0.000 & 0.000 & 0.009 & 0.009 & 0.001
\end{tabular}

c) Unemployment

\begin{tabular}{lcccccc} 
& $(1)$ & $(2)$ & $(3)$ & $(4)$ & $(5)$ & $(6)$ \\
\hline Average Absolute & -0.051 & -0.087 & -0.056 & & & \\
Point Disagreement & $(0.072)$ & $(0.094)$ & $(0.051)$ & & & \\
Average Absolute & & & & $0.570^{* * *}$ & $0.668^{* * *}$ & $0.325^{* *}$ \\
Density Disagreement & & & & $(0.198)$ & $(0.231)$ & $(0.179)$ \\
Constant & $0.819^{* * *}$ & $0.818^{* * *}$ & $0.644^{* * *}$ & $0.557^{* * *}$ & $0.446^{* * *}$ & $0.487^{* * *}$ \\
& $(0.053)$ & $(0.066)$ & $(0.051)$ & $(0.076)$ & $(0.109)$ & $(0.099)$ \\
\hline Time effects & $\mathrm{N}$ & $\mathrm{Y}$ & $\mathrm{Y}$ & $\mathrm{N}$ & $\mathrm{Y}$ & $\mathrm{Y}$ \\
Respondent effects & $\mathrm{N}$ & $\mathrm{N}$ & $\mathrm{Y}$ & $\mathrm{N}$ & $\mathrm{N}$ & $\mathrm{Y}$ \\
Incremental R & 0.001 & 0.002 & 0.001 & 0.086 & 0.097 & 0.018 \\
\hline
\end{tabular}

Notes: Standard errors are reported in parentheses and are based on clustering at the respondent level. The incremental $R^{2}$ pertains to the disagreement variable. For specifications (1) and (4), the incremental $\mathrm{R}^{2}$ is identical to the overall $\mathrm{R}^{2}$.

*** significant at the $1 \%$ level

${ }^{* *}$ significant at the $5 \%$ level

* significant at the 10\% level 
Figure 1. Motivating a Density Disagreement Measure
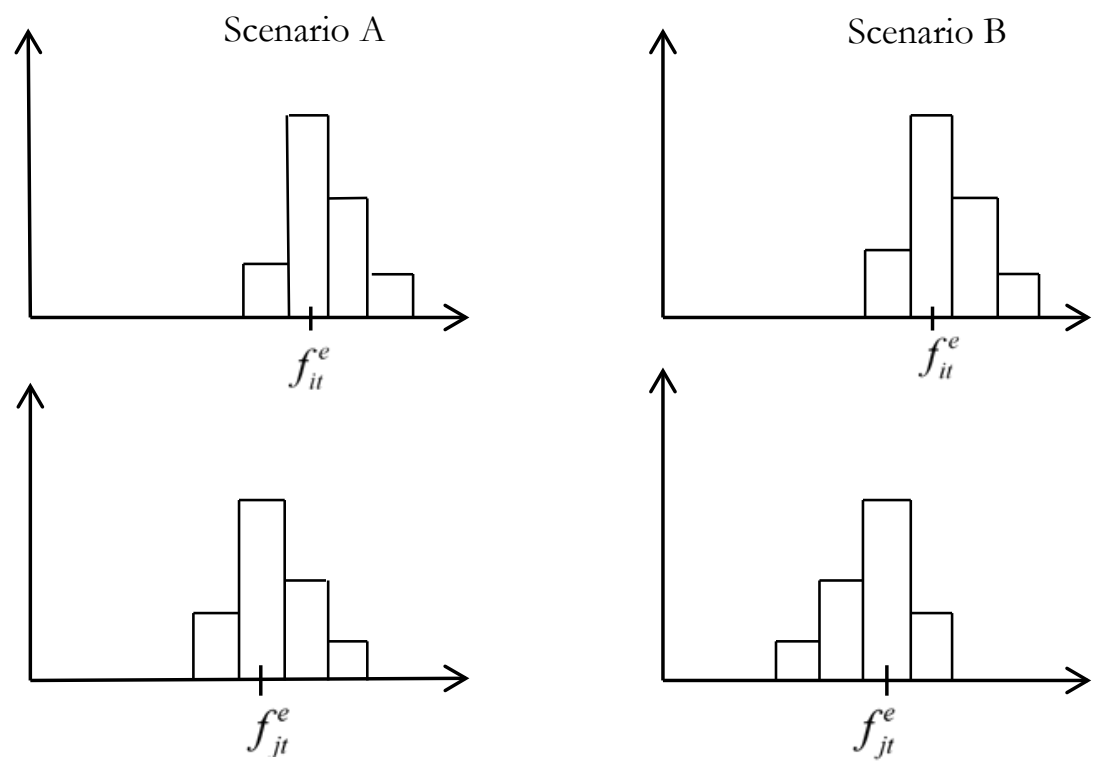
Figure 2. Individual Point- and Density-based Measures of Disagreement

a) Real GDP Growth

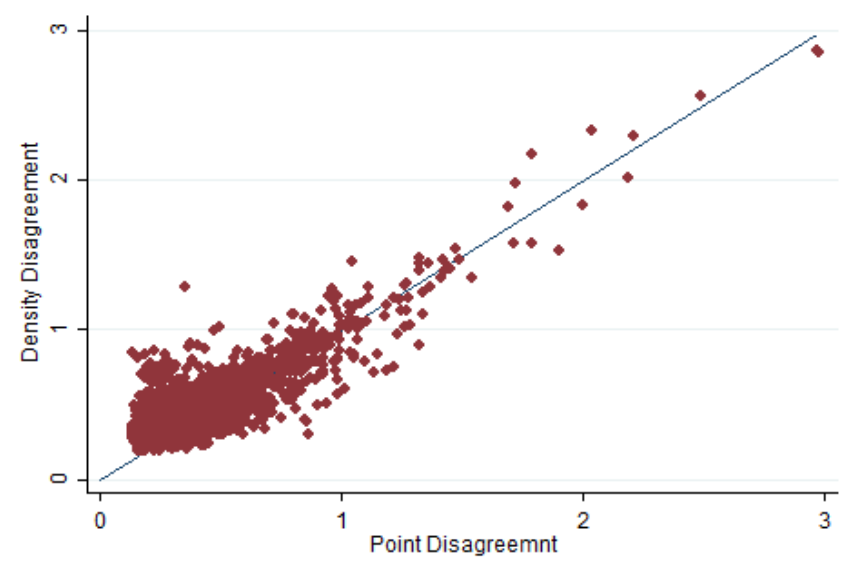

b) Inflation

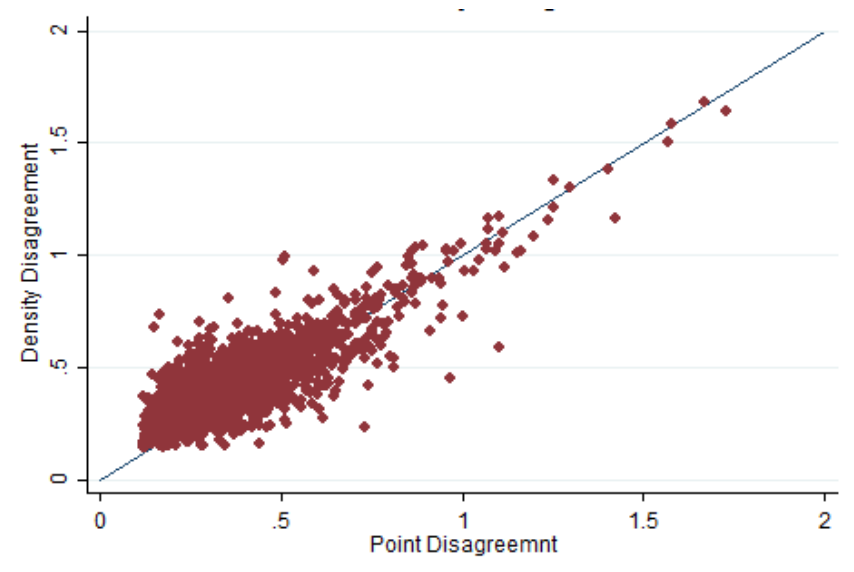

c) Unemployment

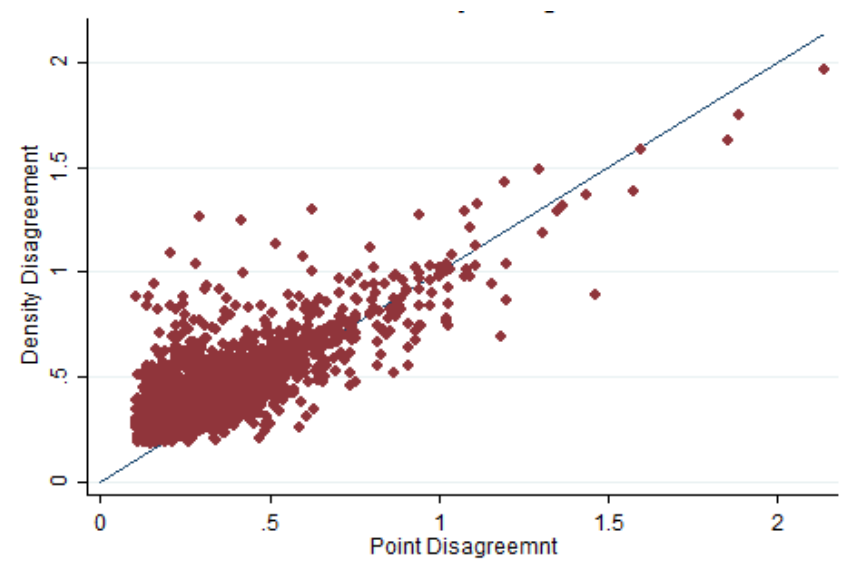


Figure 3. Distribution of Individual Disagreement Over Time

a) Real GDP Growth
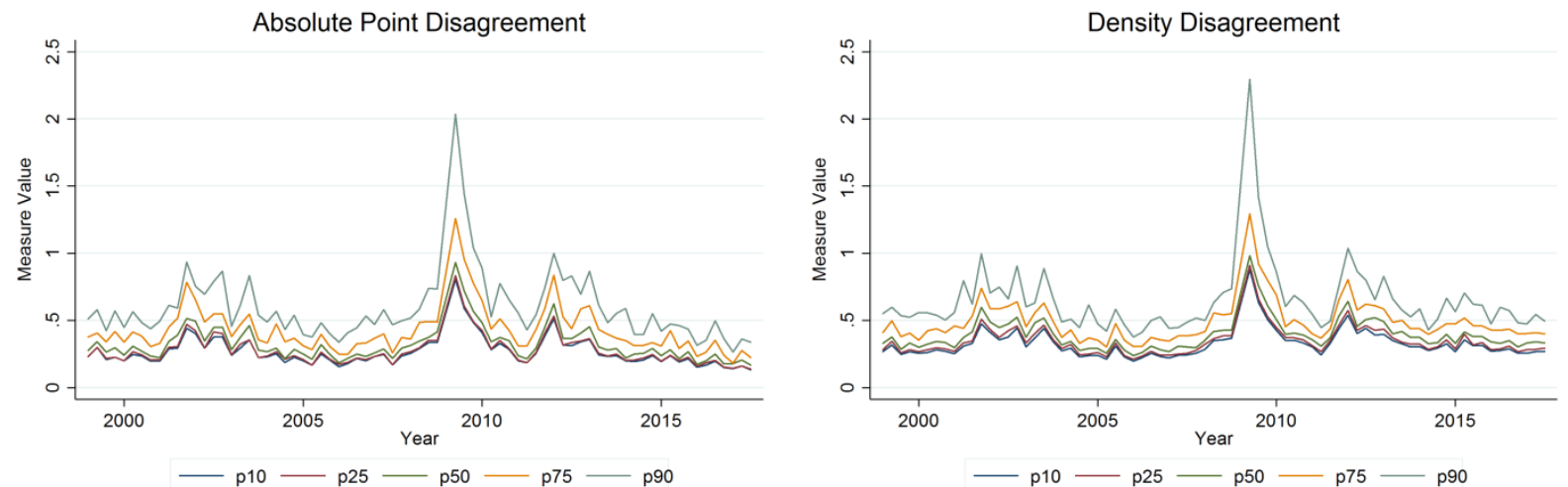

b) Inflation
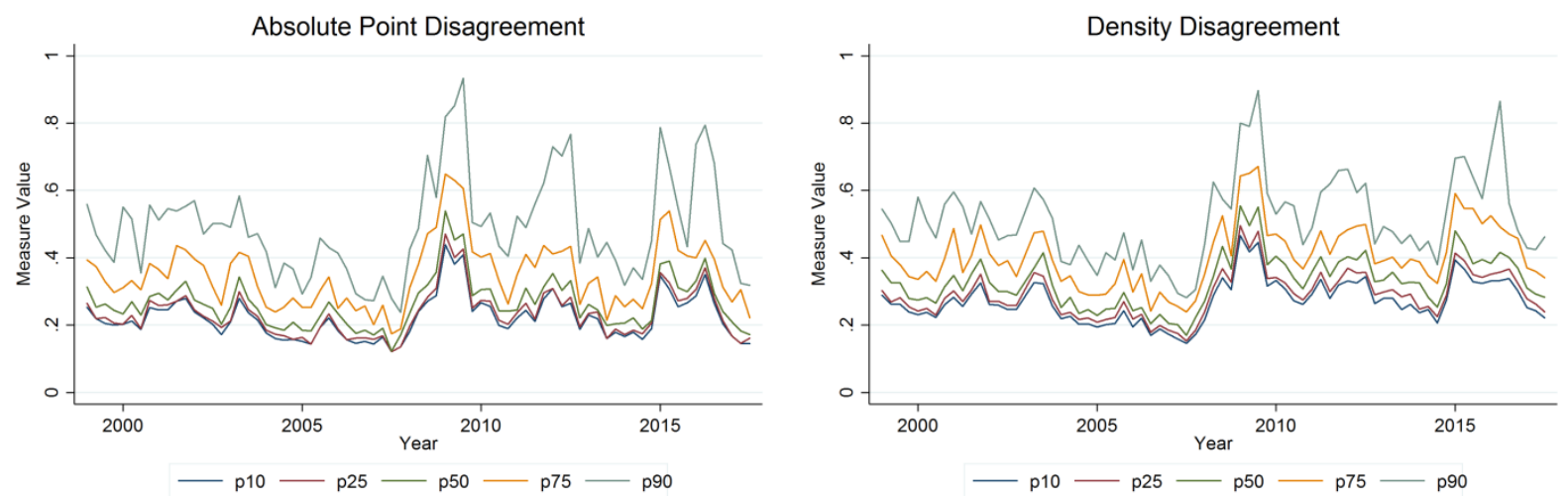

c) Unemployment
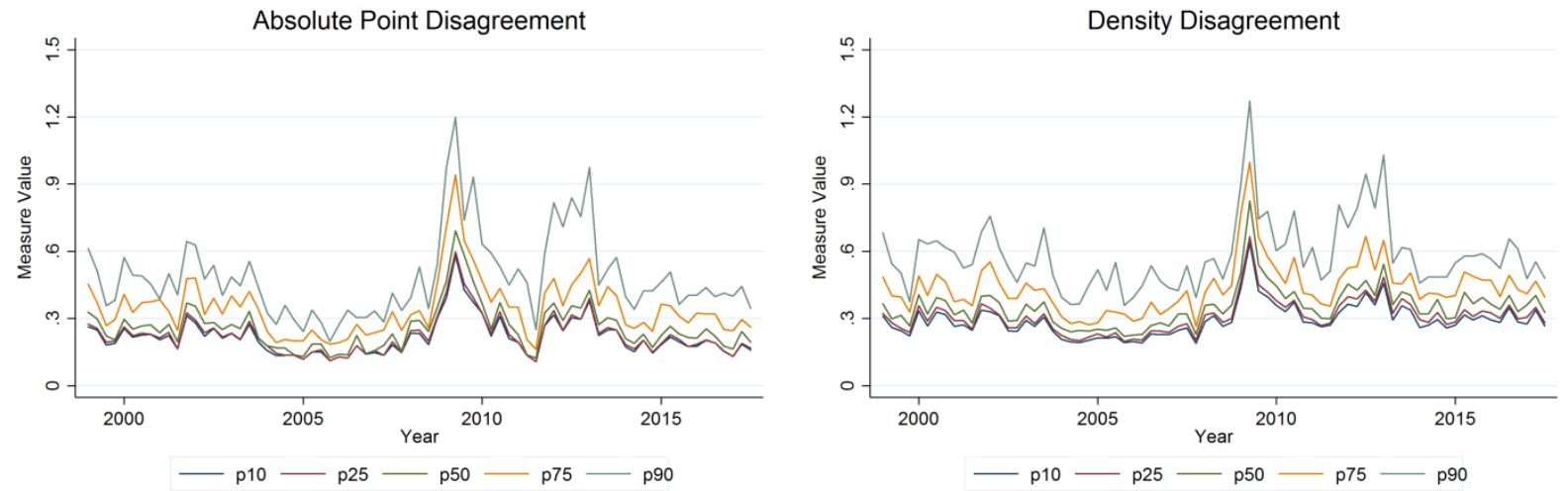
Figure 4. Distribution of Individual Uncertainty (IQR) Over Time

a) Real GDP Growth

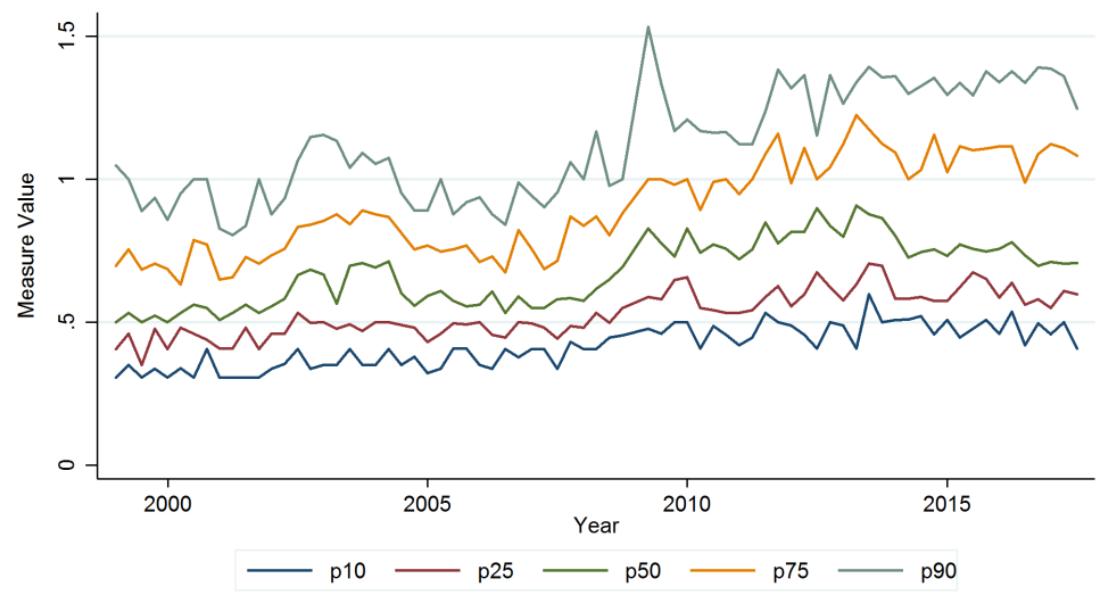

b) Inflation

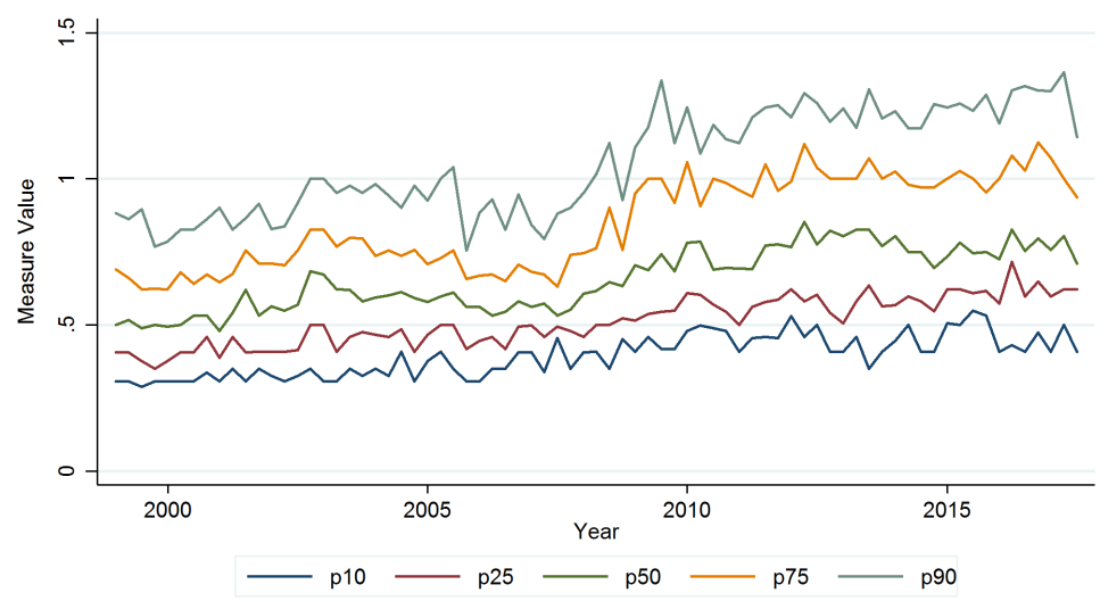

c) Unemployment

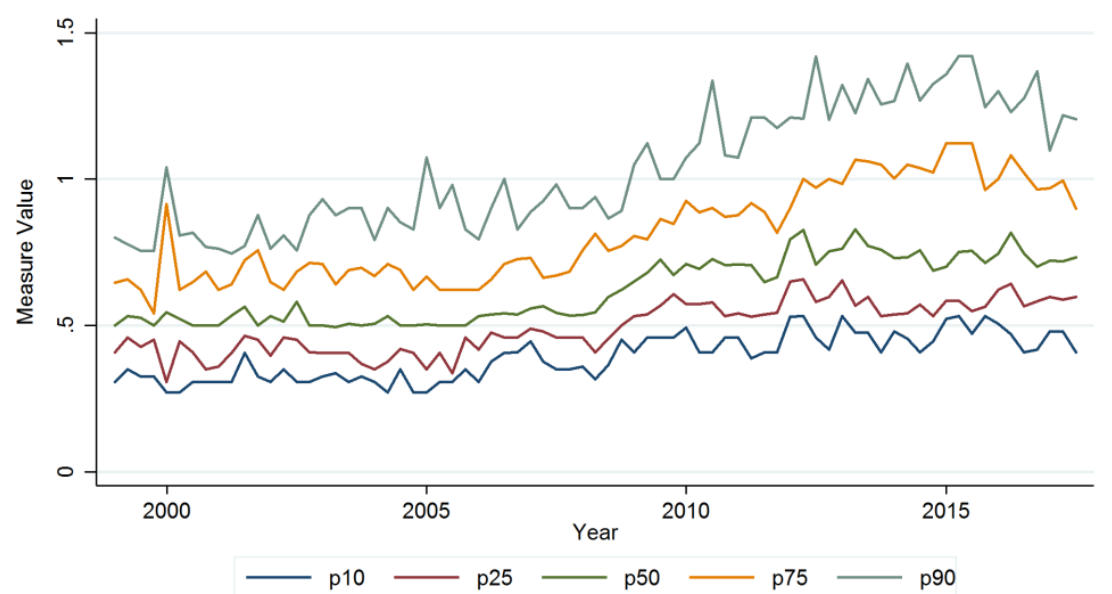

